\title{
Análisis genético de las mutaciones presentes en las poblaciones virales en pacientes con infección por VIH-1 en Ecuador
}

\begin{abstract}
Manuel González-González, Consuelo Correa-Sierra, Katherine Hermida-Álava, Ana Machado-Díaz, L. Fernando Gómez-Andrade, Martha Castillo-Segovia, C. Lissette Pérez-Santos y Vivian Kourí-Cardellá
\end{abstract}

\section{Genetic analysis of the mutations in HIV-1 infected population in Ecuador}

Background: The international recommendations of antiretroviral treatment include resistance tests to guide the treatment regimen in each patient, which is not available on a regular basis in Ecuador. Aim: To describe mutations that confer resistance to antiretrovirals in a population of Ecuadorian patients. Methods: Plasma samples from $101 \mathrm{HIV}-1$ patients with failure to antiretroviral therapy, divided into 15 children and 86 adults, were studied with the GS Junior (Roche) and the sequences were analyzed with the DeepChek program. Results: The most frequent mutations were M184V/I, K101E/P/H, K103N/S, D30N, M46L/I, I54L/M, V82T/F/A/S/L and L90M in adults and F77L, K103N/S, M46L/I, V82T/F/A/S/L and L90M in children. High resistance to non-nucleoside reverse transcriptase (RT) inhibitors in minority viral populations of adults and children $(34.9 \%$ and $70 \%)$ was detected; in children both viral populations (majority and minority viral populations) ( $>45 \%$ ) were protease inhibitor resistant. Patients who had a greater number of therapeutic regimens had higher levels of resistance to antiretrovirals. Most of the samples were subtype B in the TR and protease region, and CRF25_cpx in integrase. Conclusions: Mutations and resistance to antiretrovirals are shown in a population of Ecuadorian patients with HIV-1. These results will make it possible to issue a warning to health authorities about the need for resistance studies.

Key words: Ecuador, HIV, resistance, antiretroviral, mutations, subtypes.

Palabras clave: Ecuador, VIH, resistencia, anti-retroviral, mutaciones, subtipos.

\section{Introducción}

os anti-retrovirales (ARV) deben administrarse de por vida en los pacientes con SIDA y existen una serie de factores que pueden producir un impacto negativo en el éxito del tratamiento. El desarrollo de cepas virales resistentes es una de las principales razones del fracaso de la terapia $\mathrm{ARV}^{1}$, debido a que si se acumulan mutaciones de resistencia a muchas clases de fármacos se limita el número de alternativas de regímenes terapéuticos y el éxito virológico de las terapias siguientes.

Se dispone de métodos genotípicos y fenotípicos para determinar la resistencia ARV. El ensayo genotípico es la técnica más difundida, más rápida, de menor complejidad técnica, menor costo económico y presenta la ventaja adicional de que las mutaciones que se detectan pueden preceder a las resistencias fenotípicas ${ }^{1}$.

En Ecuador, según los últimos datos oficiales, existen alrededor de 29.000 pacientes infectados con $\mathrm{VIH}^{2}$ y se dispone de un arsenal de 15 fármacos ARV diferentes. Los fármacos de primera línea recomendados por el programa de VIH/SIDA en el país son dos inhibidores de la transcriptasa reversa análogos de nucleósidos (ITRAN) y un inhibidor de la transcriptasa reversa no análogo nucleósido (ITRNAN), siendo el esquema más utilizado tenofovir (TDF)/emtricitabina (FTC)/efavirenz $(E F V)^{3}$. Según reportes del Ministerio de Salud, a fines del año 2014 existían alrededor de 6.765 pacientes con tratamiento ARV y se estima que cada año, alrededor de 300 pacientes necesitan un cambio en la terapia ${ }^{4}$.

Las guías internacionales actuales de tratamiento ARV incluyen el empleo de las pruebas de resistencia con el fin de guiar a los médicos en la toma adecuada de decisiones respecto a los regímenes de tratamiento en cada caso ${ }^{5}$; estas pruebas desafortunadamente no están disponibles de forma estable en Ecuador.

Por ello nos trazamos los siguientes objetivos: Determinar las mutaciones que confieren resistencia a los ARV presentes en las poblaciones virales, así como los niveles de resistencia y las variantes genéticas del VIH-1, en una población de pacientes ecuatorianos con fallo a la terapia ARV.

\section{Materiales y Métodos}

Se realizó un estudio observacional, descriptivo, de corte transversal. El universo del estudio estuvo consti-
Facultad de Ciencias Médicas, Universidad de Guayaquil, Ecuador. Instituto Nacional de Investigación en Salud Pública "Leopoldo Izquieta Pérez". Dirección de Transferencia Tecnológica (MGG). Departamento de Investigación, Desarrollo e Innovación (CCS, KHA, AMD, MCS).

Centro Nacional de Referencia de Retrovirus, Hepatitis y otros Virus de Transmisión Sexual (LFGA). Instituto de Medicina Tropical Pedro Kourí, Cuba.

Laboratorio de Infecciones de Transmisión Sexual, Departamento de Virología (LPS, VKC, CLPS).

El presente estudio se realizó en el Instituto Nacional de Investigación en Salud Pública "Leopoldo Izquieta Pérez", Ecuador.

Los autores declaran que no hay conflicto de interés.

Fuente de financiamiento: SENESCYT, a través del proyecto INSPI-ESPOL titulado "Epidemiología Molecular del VIH-1 en el Ecuador".

Recibido: 16 de enero de 2017 Aceptado: 5 de enero de 2018

Correspondencia a: Consuelo Correa-Sierra consuelocorreasierra@gmail.com 
tuido por todas las muestras de plasma de los pacientes con infección por VIH-1 recibidas en el Laboratorio de Virología del Instituto Nacional de Investigación en Salud Pública "Leopoldo Izquieta Pérez" (INSPI), Guayaquil, junto a su respectiva ficha de datos epidemiológicos, como parte del diagnóstico y seguimiento del Programa Nacional de Control y Prevención del VIH/SIDA. El período de estudio abarcó desde mayo de 2013 a diciembre de 2015. La muestra quedó comprendida por 101 plasmas de pacientes que cumplieron los criterios de inclusión.

Los criterios de inclusión fueron: muestras de plasma que tuvieran como mínimo $1 \mathrm{~mL}$, que hubieran estado conservadas a $-80^{\circ} \mathrm{C}$ y cuya carga viral $(\mathrm{CV})$ previa fuese mayor a 2.000 copias $/ \mathrm{mL}$ para las muestras recibidas entre mayo de 2013 y febrero de 2014 (74 plasmas) o 20.000 copias $/ \mathrm{mL}$ para las muestras recibidas luego de febrero de 2014 ( 27 plasmas). La pirosecuenciación 454 se descontinuó y se emplearon los reactivos disponibles en el mercado compatibles con el secuenciador utilizado; por esta razón se emplearon dos versiones (2.0 y 3.0) del sistema de pirosecuenciación 454 , con diferentes sensibilidades (20.000 y 2.000 copias $/ \mathrm{mL})$. Las muestras corresponden a pacientes con el antecedente de haber sido tratados con ARV y tener fallo a la terapia ARV (virológico, clínico o inmunológico, según lo definido en la literatura médica $)^{6}$.

El criterio considerado como CV mínima se seleccionó en base al límite de detección de la técnica empleada, a pesar de que puede haber pacientes con fallo a la terapia ARV y carga viral $<2.000$ copias ARN/mL.

Las muestras de plasma procesadas provenían de las clínicas de SIDA de diferentes hospitales de las provincias de Pichincha, Guayas, Esmeraldas, Los Ríos y El Oro. El presente estudio forma parte de los resultados del proyecto INSPI-ESPOL "Epidemiología Molecular del VIH-1 en el Ecuador", auspiciado por SENESCYT. Veintisiete de las muestras incluidas en el presente estudio forman parte de una publicación reciente en Revista Cubana de Medicina Tropical ${ }^{7}$.

El estudio contó con la aprobación del Comité Científico institucional.

\section{Experimentos realizados}

La pirosecuenciación se seleccionó como parte de un proyecto de investigación, en el que se consideró esta metodología como prominente y apoyado por la sencillez del software para el análisis de los datos en el momento de su diseño experimental.

Las muestras fueron procesadas a través del sistema de pirosecuenciación 454 de ROCHE para el gen pol del VIH-1. A continuación se describe brevemente la metodología empleada.

La extracción del ARN del VIH se realizó con el estuche High Pure Viral Nucleic Acid Large Volume (Roche,
EUA), siguiendo las recomendaciones del fabricante. Luego se procedió a la transcripción reversa, síntesis de ADN complementario, generación de amplicones, purificación de los productos de la reacción de polimerasa en cadena (RPC), cuantificación de los productos purificados de RPC, elaboración de la librería de amplicones, RPC en emulsión, recuperación de las perlas con los productos de RPC obtenidos, cuantificación de las perlas recuperadas y la secuenciación, utilizando las especificaciones detalladas en el manual GS Junior Training (Roche) $)^{8}$ y las instrucciones del fabricante (Roche) $)^{9,10}$.

En 27 muestras se utilizó la versión 2.0 que permite la detección de fragmentos del gen pol que codifican para la transcriptasa reversa (TR) y la proteasa (PR) con un límite de detección de 20.000 copias $/ \mathrm{mL}^{11}$, en el resto de las muestras ( 74 plasmas) se utilizó la versión 3.0 en la que además de los fragmentos anteriores se detecta el fragmento que codifica la integrasa (IN) y posee un límite de detección de 2.000 copias $/ \mathrm{mL}^{12}$.

Las secuencias resultantes fueron editadas automáticamente por el software DeepChek 1.3 (Advanced Biological Laboratories).

\section{Análisis de la resistencia}

Para la clasificación de la resistencia se siguió el algoritmo de Stanford, versión 7.0 (27/2/2014), http:// hivdb.stanford.edu/pages/documentPage/drm.html) y para el análisis de las mutaciones se incluyeron aquellas internacionalmente reconocidas y en la región de la PR sólo se incluyeron las mutaciones mayores ${ }^{13}$. Las secuencias fueron ingresadas al software DeepCheck-HIV algorithms entre julio y diciembre de 2015. Los niveles de resistencia definidos por el DeepChek-HIV algorithms v1.3 para la base de datos de Stanford (v7.0- 27/2/2014) fueron: categoría susceptible $(\mathrm{S})$, resistencia intermedia (RI) y la resistencia alta (RA). Los niveles de resistencia a los tres grupos de medicamentos se calcularon mediante la determinación del promedio del porcentaje de RA, RI y $\mathrm{S}$ de cada medicamento individual que pertenece a cada clase de ARV. Se consideró que las muestras de los pacientes tenían resistencia cuando presentaron RI o RA. Se clasificó como resistencia a todos los fármacos de una clase (del inglés full class resistance o FCR) cuando no existía ningún ARV sensible a la clase en cuestión ${ }^{14}$. La multi-resistencia o resistencia a todas las clases de fármacos estudiados (del inglés multidrug resistance o MDR) se definió como los virus resistentes a todos los medicamentos de las tres clases, pudiendo encontrarse un solo medicamento de una clase sensible ${ }^{14}$.

\section{Análisis estadístico}

Se determinaron las frecuencias absolutas y relativas de las variables estudiadas. Las variables incluyeron las mutaciones que confieren resistencia a los ARV en 
las poblaciones virales minoritarias, en las poblaciones mayoritarias y en su conjunto.

Se define como poblaciones mayoritarias virales aquellas que están presentes en más de $20 \%$ de los virus analizados y poblaciones minoritarias virales como aquellas que están presentes en el rango de 1 a $20 \%$ de los virus analizados en una muestra.

Los datos recogidos abarcaron las siguientes variables de estudio: edad (en años cumplidos), sexo (femenino o masculino), preferencia sexual (heterosexual, homosexual o bisexual), terapia anti-retroviral (medicamentos, número de terapias), valor de la última $\mathrm{CV}$ y valor del último recuento de linfocitos (LT) T CD4+. Las variables cuantitativas continuas (edad, CV y recuento de LT CD4) fueron analizadas también a través de la mediana, considerando los valores del $1^{\text {er }}$ y $3^{\text {er }}$ cuartil. El análisis entre los niveles de resistencia o mutaciones y el antecedente del empleo de ARV se realizó considerando solamente a los pacientes con el dato del tipo de terapia ARV utilizada.

Se realizó una regresión logística para buscar la posible asociación entre los subtipos y las mutaciones encontradas.

\section{Resultados}

Las características de la muestra estudiada se pueden apreciar en la Tabla 1.

\section{Niveles de resistencia a los medicamentos anti-retrovirales}

De forma general, los niveles de resistencia a los ARV fueron mayores en las poblaciones virales minoritarias al compararlos con los niveles de resistencia de las poblaciones virales mayoritarias.

Los niveles de resistencia de los virus frente a los ITRNAN y los inhibidores de proteasa (IP) fueron los más altos en ambas poblaciones virales de las muestras de los adultos, (mayoritarias 34,9 y $29,1 \%$, y minoritarias 47,7 y $68,6 \%$, respectivamente, Figura 1 A), particularmente para los medicamentos efavirenz (EFV) y nevirapina (NVP) en el grupo de los ITRNAN, y para atazanavir (ATZ)/ potenciado con ritonavir (/r), lopinavir (LPV/r), nelfinavir (NFV), fosamprenavir (FPV/r), indinavir (IDV/r) y saquinavir (SQV/r) (Figura $1 \mathrm{~B})$.

En poblaciones virales mayoritarias de los adultos, se encontraron con FCR dos muestras $(2,3 \%)$ a los ITRAN, $12(13,9 \%)$ a ITRNAN, ninguna a IP y dos a inhibidores de la integrasa IN $(3,1 \%)$. En poblaciones minoritarias, se encontraron cuatro muestras con FCR a los ITRAN (4,7\%), 16 a los ITRNAN (18,6\%), cinco a los IP (5,8\%) y dos a IIN $(3,1 \%)$; adicionalmente, una muestra $(1,2 \%)$ tuvo MDR (sin incluir a IIN).

En las poblaciones virales mayoritarias de los niños, se encontró una elevada resistencia a los IP $(46,7 \%)$. La resistencia a los IIN $(33,3 \%)$, debe ser analizada cuidadosamente pues sólo tres niños tuvieron virus analizados en

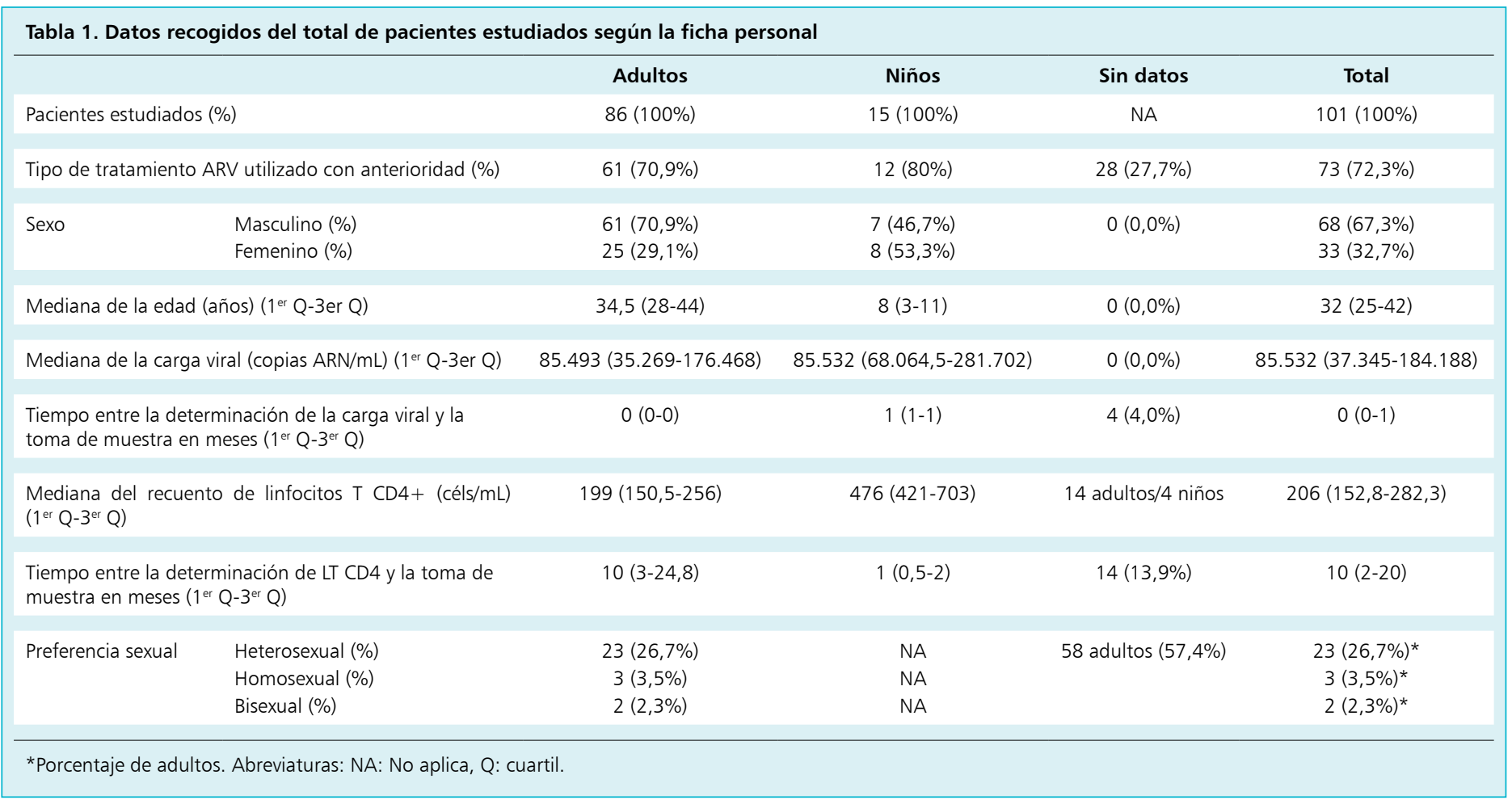




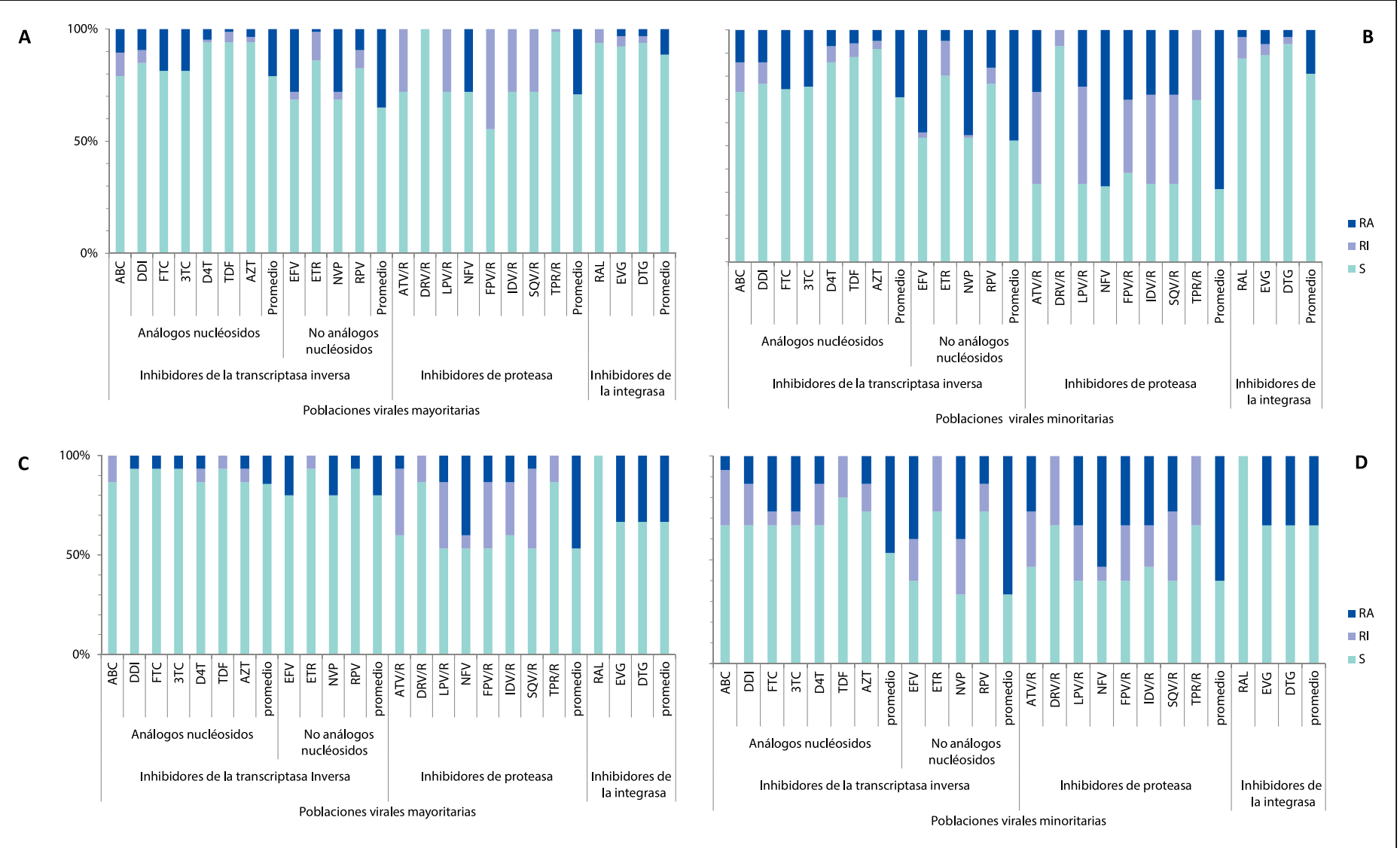

Figura 1. Niveles de resistencia a los ITRAN, ITRNAN e IP en poblaciones mayoritarias y minoritarias de VIH-1 en pacientes adultos (A y B) y niños (C y D). Abreviaturas: ABC: Abacavir, AZT: zidovudina, ATV: atazanavir, DRV: darunavir, d4T: estavudina, ddC: zalcitabina, ddl: didanosina, DTG: dolutegravir, EFV: efavirenz, ETR: etravirine, EVG: elvitegravir, FTC: emtricitabina, FPV: fosamprenavir, IDV: indinavir, LPV: lopinavir, NFV: nelfinavir, NVP: nevirapina, RA: resistencia alta, RAL: raltegravir, RI: resistencia intermedia, RPV: rilpivirina, S: susceptible, SQV: saquinavir, TDF: tenofovir, TPR: tipranavir, 3TC: lamivudina, /R: potenciado con ritonavir.

la región de la IN y uno de ellos (en el que se desconoce el antecedente de empleo de este grupo de medicamentos) presentó resistencia a los IIN. Los medicamentos con mayores niveles de resistencia en poblaciones mayoritarias fueron los IP (exceptuando DRV/r y tripanavir (TPR/r), elvitegravir (EVG) y dolutegravir (DTG) (Figura $1 \mathrm{C}$ ). En poblaciones virales minoritarias se encontró que los niveles de resistencia a todas las clases de medicamentos ARV fueron altos, particularmente para los medicamentos EFV, NVP (40,0\%) y los IP (Figura 1 D). En poblaciones minoritarias se encontraron tres muestras con FCR a los ITRNAN, uno a los ITRAN y tres a los IP; mientras que en las poblaciones virales mayoritarias se encontró una muestra con FCR a cada una de las clases de ARV estudiadas. Se detectaron virus MDR en poblaciones minoritarias en un niño de un año de edad.

\section{Análisis de mutaciones que confieren resistencia a anti-retroviral en los genes de la transcriptasa reversa, proteasa viral e integrasa}

Provenientes de los adultos, se encontraron muestras con un total de 23 mutaciones en el gen de la TR que confieren resistencia a los ARV, 11 de ellas a uno o varios de los medicamentos de la familia de los ITRAN y 12 mutaciones a uno o varios de los medicamentos de la familia de los ITRNAN. Además, se detectaron nueve mutaciones mayores, que confieren resistencia a uno o varios de los medicamentos de la familia de los IP (Figura 2 A).

Las mutaciones más frecuentes encontradas en la región de la TR que confieren resistencia a los ITRAN y los ITRNAN, fueron: M184V/I (18,6\%), K101E/P/H $(17,4 \%)$ y K103N/S (25,6\%, Figura 4 A). Entre las mutaciones primarias asociadas a resistencia en la región de la PR prevalentes, se encontraron: D30N (22,1\%), M46L/I $(27,9 \%)$, I54L/M (18,6\%), V82T/F/A/S/L (25,6\%) у L90M (36\%).

Se detectaron diferencias en la frecuencia de mutaciones que confieren resistencia en poblaciones virales minoritarias y mayoritarias. Por ejemplo, en poblaciones 


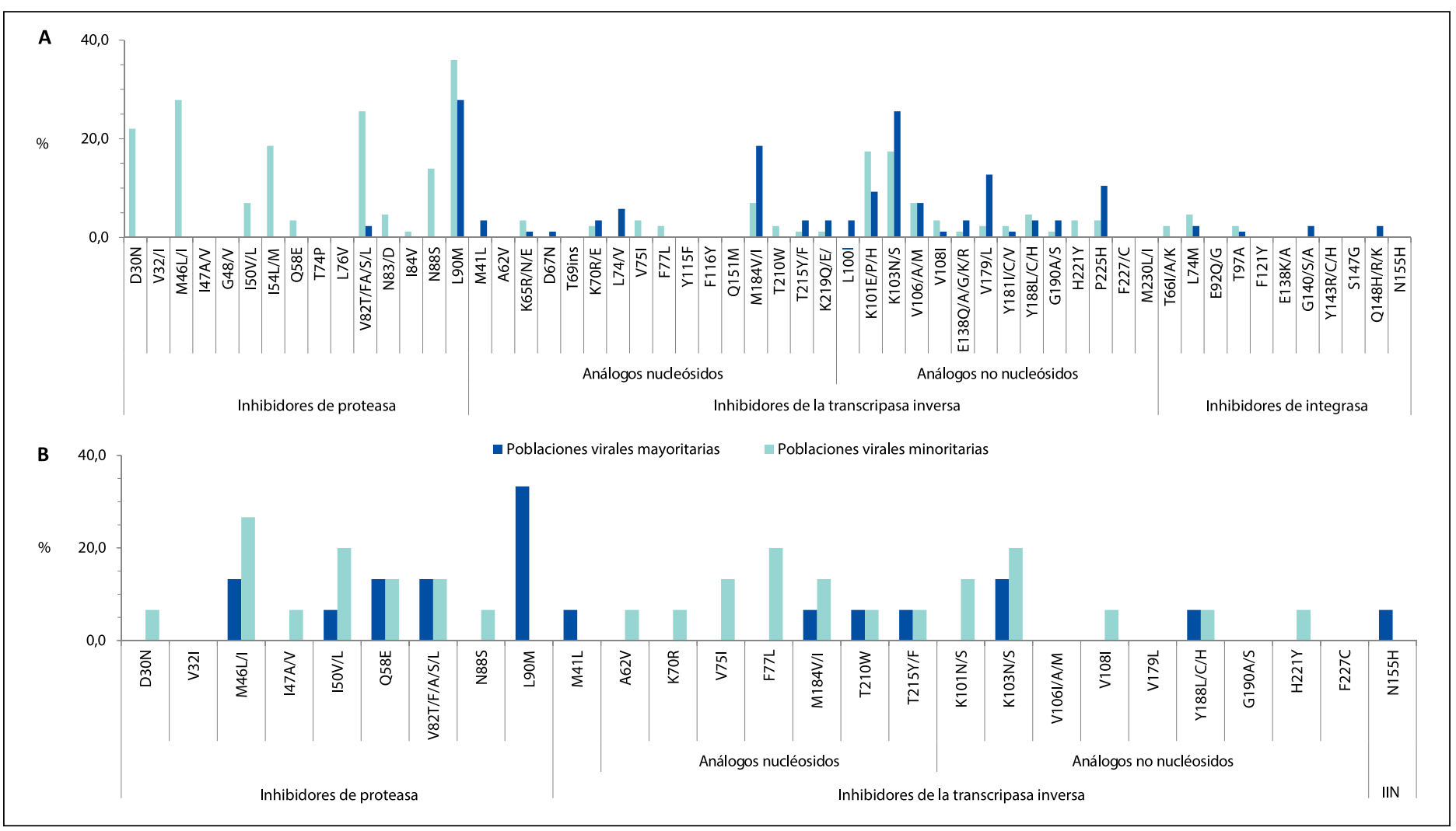

Figura 2. Porcentaje de pacientes adultos con poblaciones de $\mathrm{VIH}-1$ con mutaciones que confieren resistencia a los ARV en poblaciones minoritarias ( $<20 \%)$ y mayoritarias (> 20\%). A: adultos y B: niños. Abreviatura: IIN: inhibidores de integrasa.

mayoritarias se hallaron mutaciones menos frecuentes, pero de gran importancia como la M41L, K70R, L74V, $\mathrm{T} 215 \mathrm{~F} / \mathrm{Y}, \mathrm{K} 219 \mathrm{Q} / \mathrm{E}, \mathrm{L} 100 \mathrm{I}, \mathrm{V} 106 \mathrm{~A} / \mathrm{M}, \mathrm{E} 138 \mathrm{Q} / \mathrm{A} / \mathrm{G} / \mathrm{K} / \mathrm{r}$ V179L, Y188L/C/H, G190A/S y P225H.

En las muestras provenientes de niños, la mutación detectada con mayor frecuencia en poblaciones virales mayoritarias en la región de la PR fue la L90M $(33,3 \%)$, mientras que en la región de la TR fue la K103N/S $(13,3 \%)$ y en la región de la IN la N155H (6,7\%). En poblaciones minoritarias virales las mutaciones más detectadas fueron M46L/I $(25,7 \%), \mathrm{I} 50 \mathrm{~V} / \mathrm{L}(20,0 \%)$ en la región de la PR y la F77L (20,0\%), K103N/S en la región de la TR $(20,0 \%$, Figura $2 \mathrm{~B})$.

La frecuencia de mutaciones que confieren resistencia encontradas en poblaciones mayoritarias $(>20 \%)$ es menor a la frecuencia de mutaciones en población minoritarias (1-20\%) (Figura 2 A y B).

\section{Niveles de resistencia a los medicamentos anti-retrovirales, mutaciones y su relación con el tratamiento empleado}

Se emplearon 17 esquemas terapéuticos en 61 pacientes adultos (Tabla 2). El tiempo de tratamiento ARV de los pacientes adultos fue variado (mediana del tiempo de inicio del tratamiento ARV: 54 meses, $1^{\text {er }}$ IQ: 44 meses- $3^{\text {er }}$ IQ: 120 meses).

Se emplearon ocho esquemas terapéuticos en 12 niños (Tabla 2). El tiempo de tratamiento ARV de los pacientes fue mayor a cuatro años en la mayoría de los casos (mediana del tiempo de inicio del tratamiento ARV: 56 meses, $1^{\text {er }}$ IQ: 34 meses- $3^{\text {er }}$ IQ: 97 meses). No se encontró relación en cuanto al tiempo de tratamiento y la presencia de resistencia a los ARV en adultos ni en niños (datos no mostrados).

De forma general, los pacientes que tuvieron un mayor número de esquemas terapéuticos presentaron mayores niveles de resistencia a los ARV, tanto en poblaciones virales mayoritarias como en las minoritarias (Figuras 3 y 4). Se detectaron algunas excepciones, en los adultos: los niveles de resistencia en los ITRNAN en las poblaciones virales mayoritarias de los pacientes con dos esquemas, fue superior a la de los pacientes con tres esquemas. Los niveles de resistencia a los IP en poblaciones virales minoritarias de pacientes que habían utilizado un solo esquema de tratamiento o que se desconocía cuantos esquemas terapéuticos habían utilizado, fueron muy altos $(74,3$ y 


\begin{tabular}{|c|c|c|c|c|}
\hline Esquema terapéutico & $\begin{array}{l}\text { Primera combinación } \\
\text { Adultos (Niños) }\end{array}$ & $\begin{array}{l}\text { Segunda combinación } \\
\text { Adultos (Niños) }\end{array}$ & $\begin{array}{l}\text { Tercera combinación } \\
\text { Adultos (Niños) }\end{array}$ & $\begin{array}{c}\text { Total } \\
\text { Adultos (Niños) }\end{array}$ \\
\hline $\mathrm{AZT}+3 \mathrm{TC}+\mathrm{EFV}$ & $16(4)$ & $1(3)$ & $0(0)$ & $17(7)$ \\
\hline $\mathrm{AZT}+3 \mathrm{TC}+\mathrm{NFV}$ & $2(4)$ & $0(1)$ & $0(0)$ & $2(5)$ \\
\hline $\mathrm{AZT}+3 \mathrm{TC}+\mathrm{LPV} / \mathrm{r}$ & $2(1)$ & $1(2)$ & $0(1)$ & $3(4)$ \\
\hline $\mathrm{TDF}+\mathrm{FTC}+\mathrm{EFV}$ & $22(0)$ & $5(0)$ & $1(0)$ & $28 \quad(0)$ \\
\hline $3 \mathrm{TC}+\mathrm{DDI}+\mathrm{EFV}$ & $1(0)$ & $0(0)$ & $0(0)$ & $1(0)$ \\
\hline $\mathrm{DDI}+3 \mathrm{TC}+\mathrm{LPV} / \mathrm{r}$ & $1(0)$ & $0(0)$ & $0(0)$ & $1(0)$ \\
\hline $\mathrm{d} 4 \mathrm{~T}+3 \mathrm{TC}+\mathrm{EFV}$ & $1(0)$ & $0(0)$ & $0(0)$ & $1(0)$ \\
\hline $3 \mathrm{TC}+\mathrm{ABC}+\mathrm{EFV}$ & $3(0)$ & $0(0)$ & $0(0)$ & $3(0)$ \\
\hline $3 T C+A B C+L P V / r$ & $2(1)$ & $3(0)$ & $1(0)$ & $6(1)$ \\
\hline $\mathrm{TDF}+\mathrm{FTC}+\mathrm{RAL}$ & $0 \quad(0)$ & $1(0)$ & $2(0)$ & $3(0)$ \\
\hline $\mathrm{TDF}+\mathrm{FTC}+\mathrm{LPV} / \mathrm{r}$ & $9(0)$ & $6(0)$ & $3(0)$ & $18 \quad(0)$ \\
\hline$A B C+E F V$ & $0 \quad(0)$ & $1(0)$ & $0(0)$ & $1(0)$ \\
\hline$A Z T+3 T C+R A L$ & $0 \quad(0)$ & $0(0)$ & $1(0)$ & $1(0)$ \\
\hline$A Z T+3 T C$ & $1(0)$ & $0(0)$ & $0(0)$ & $1(0)$ \\
\hline $\mathrm{EFV}+\mathrm{RAL}+\mathrm{DRV} / \mathrm{r}$ & $1(0)$ & $0(0)$ & $0(0)$ & $1(0)$ \\
\hline $\mathrm{DDI}+\mathrm{d} 4 \mathrm{~T}+\mathrm{LPV} / \mathrm{r}$ & $0 \quad(0)$ & $3(0)$ & $0(0)$ & $3(0)$ \\
\hline $\mathrm{FTC}+\mathrm{TDF}$ & $0(0)$ & $1(0)$ & $0(0)$ & $1(0)$ \\
\hline$A Z T+3 T C+R T V$ & 0 & $0(0)$ & $0(0)$ & 0 \\
\hline $3 T C+L P V / r$ & 0 & $0(0)$ & $0(0)$ & 0 \\
\hline$A B C+D D I+L P V / r$ & $0 \quad(0)$ & $0(1)$ & $0(1)$ & 0 \\
\hline $\mathrm{EFV}+\mathrm{ETV}$ & $0 \quad(0)$ & $0(1)$ & $0(0)$ & 0 \\
\hline $\mathrm{TDF}+\mathrm{EFV}+\mathrm{DRV} / \mathrm{r}$ & $0 \quad(0)$ & $0(0)$ & $0(1)$ & 0 \\
\hline Total & $61(12)$ & $22(8)$ & $8(3)$ & $61(12)$ \\
\hline
\end{tabular}

Abreviaturas: ABC: abacavir, AZT: zidovudina, DRV: darunavir, d4T: estavudina, DDI: didanosina, EFV: efavirenz, FTC: emtricitabina, LPV: lopinavir, NFV: nelfinavir, RAL: raltegravir, TDF: tenofovir, 3TC: lamivudina, /R: potenciado con ritonavir.

$68 \%$, respectivamente) (Figura 3 A, B y C). En los niños, la excepción encontrada: en poblaciones virales minoritarias de pacientes con dos esquemas los niveles de resistencia a los ITRNAN fue el mayor (100\%, Figura 4 A, B, C y D).

El empleo de varios esquemas terapéuticos ARV y su relación con la resistencia a los fármacos tuvo una distribución similar en poblaciones virales minoritarias y mayoritarias, teniendo en esta última una menor proporción de virus resistentes (Figura 3 y 4 A, B, C y D). No se obtuvieron los datos del tratamiento ARV empleado en 25 pacientes adultos (Figura $3 \mathrm{D}$ ) y tres niños (Figura $4 \mathrm{D}$ ).

El 98\% de los pacientes adultos utilizó ITRAN, 79\% recibió ITRNAN, 46\% IP y $8 \%$ se trató con IN; siendo los medicamentos más utilizados FTC (75\%), TDF (75\%), EFV (77\%), LPV/r (44\%) y RAL (8\%). Todos los niños habían utilizado ITRAN, 75\% ITRNAN e IP; siendo los medicamentos más utilizados 3TC (100\%), AZT (83,3\%), EFV (75\%) y LPV/r (58,3\%).

$\mathrm{Al}$ analizar la resistencia en poblaciones mayoritarias y minoritarias con respecto al antecedente del uso de cada uno de los ARV, se encontró que, de forma general, los pacientes con el antecedente de tomar medicamentos tenían virus con mayores valores de resistencia al medicamento empleado (Figura 3 y 4 E). Los niños presentaron poblaciones virales con mayores niveles de resistencia a los medicamentos ABC, DDI, DRV/r, LPV/r y NFV habiendo sido estos medicamentos utilizados en menor medida (Figura 4 E). La concordancia entre la presencia de mutaciones que confieren resistencia a los ARV y el antecedente de uso de ARV fue alta para los IP (73\%) y para los ITRNAN $(59,6 \%)$, siendo menor para los ITRAN $(41,7 \%)$ y los IIN $(20 \%)$

\section{Otras variables estudiadas}

La mediana de LTCD4+ fue baja (Tabla 1). Los recuentos de LTCD4+ menores a $200 / \mathrm{mm}^{3}$ o carga viral mayor a 100.000 copias $\mathrm{ARN} / \mathrm{mL}$ no se asociaron a mayores niveles de resistencia de forma general y para 


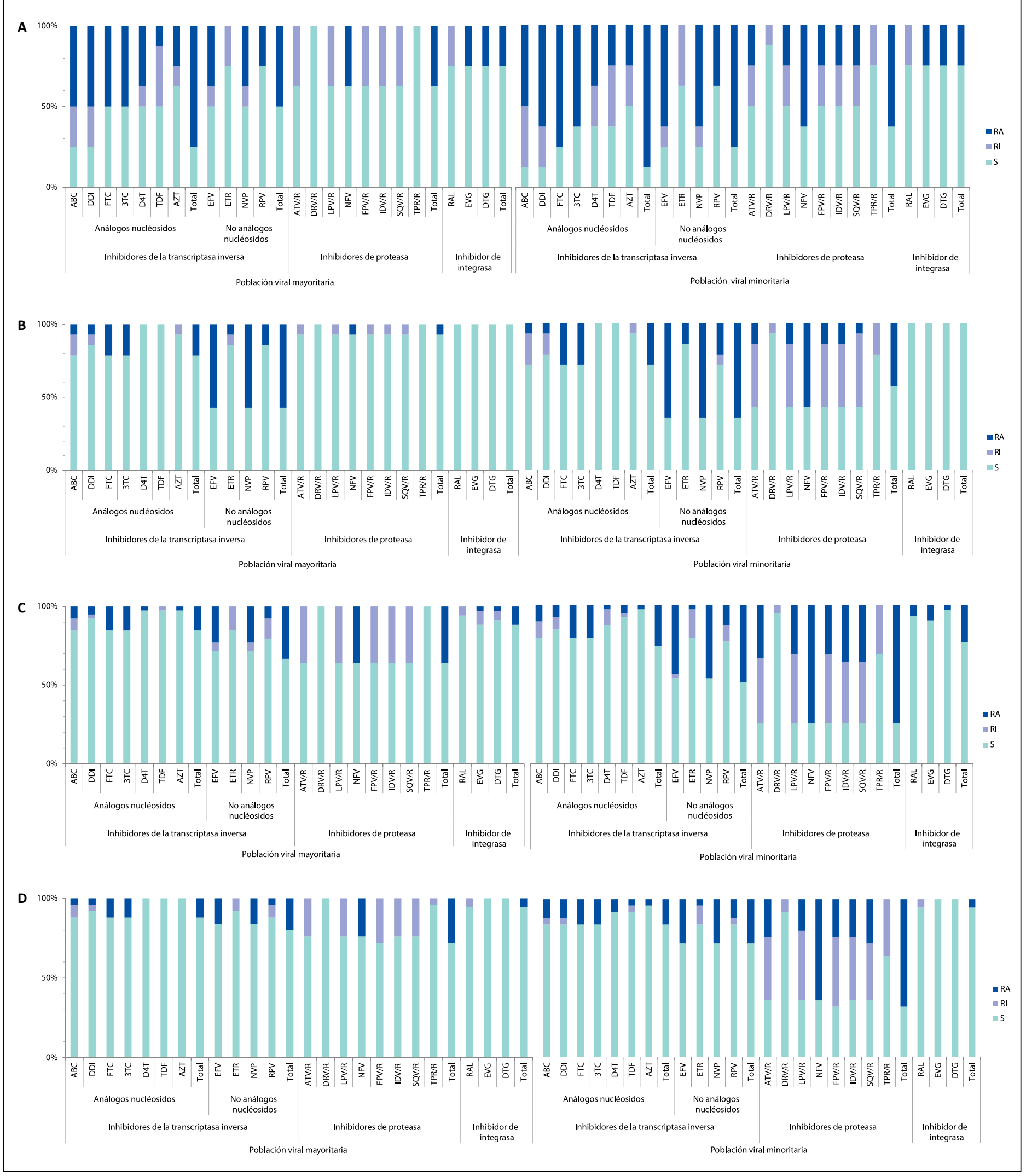




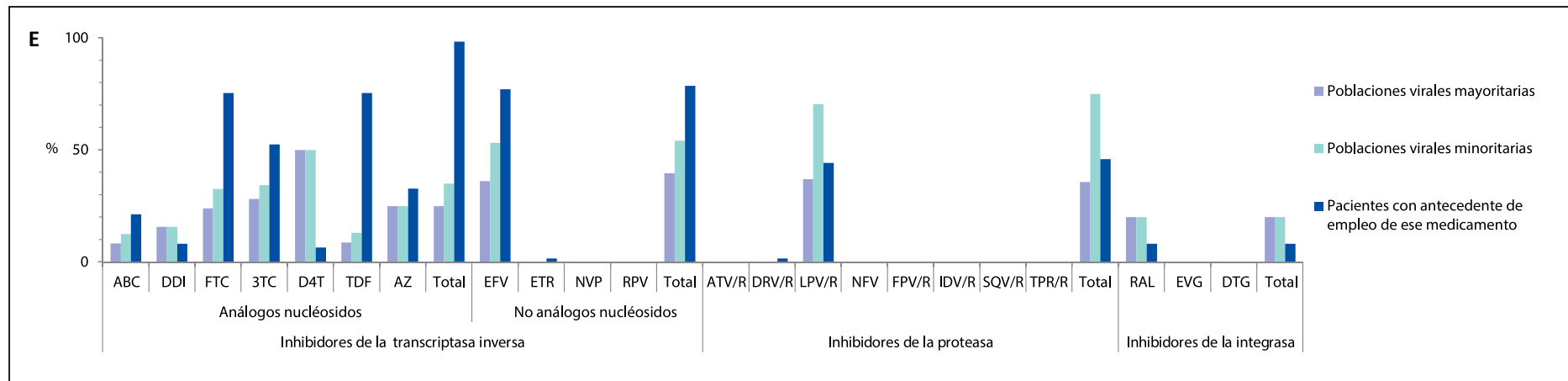

Figura 3. Porcentaje de resistencia a los antiretrovirales en poblaciones minoritarias y mayoritarias de $\mathrm{VIH}-1$ según ARV utilizados previamente en pacientes adultos (A: 3 o más esquemas terapéuticos, B: 2 esquemas terapéuticos, C: 1 esquema terapéutico, D: se desconoce cuántos esquemas terapéuticos han utilizado y E: Niveles de resistencia a los ARV y tratamiento previo con ese ARV). Abreviaturas: ABC: Abacavir, AZT: zidovudina, ATV: atazanavir, DRV: darunavir, d4T: estavudina, ddC: zalcitabina, ddl: didanosina, DTG: dolutegravir, EFV: efavirenz, ETR: etravirine, EVG: elvitegravir, FTC: Emtricitabina, FPV: fosamprenavir, IDV: indinavir, LPV: lopinavir, NFV: nelfinavir, NVP: nevirapina, RA: resistencia alta, RAL: raltegravir, RI: resistencia intermedia, RPV: rilpivirina, S: susceptible, SQV: saquinavir, TDF: tenofovir, TPR: tripanavir, 3TC: lamivudina, /R: potenciado con ritonavir.

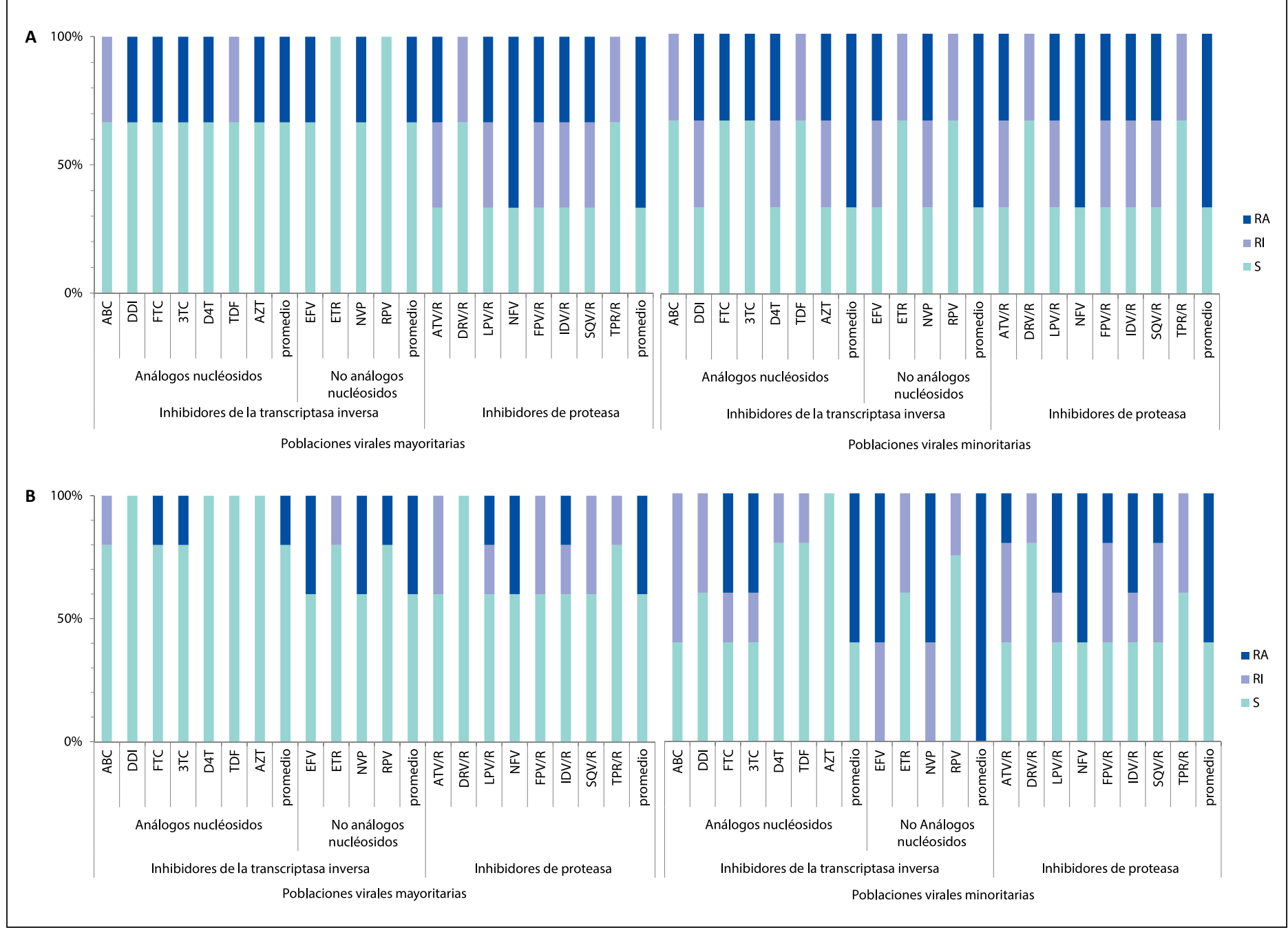




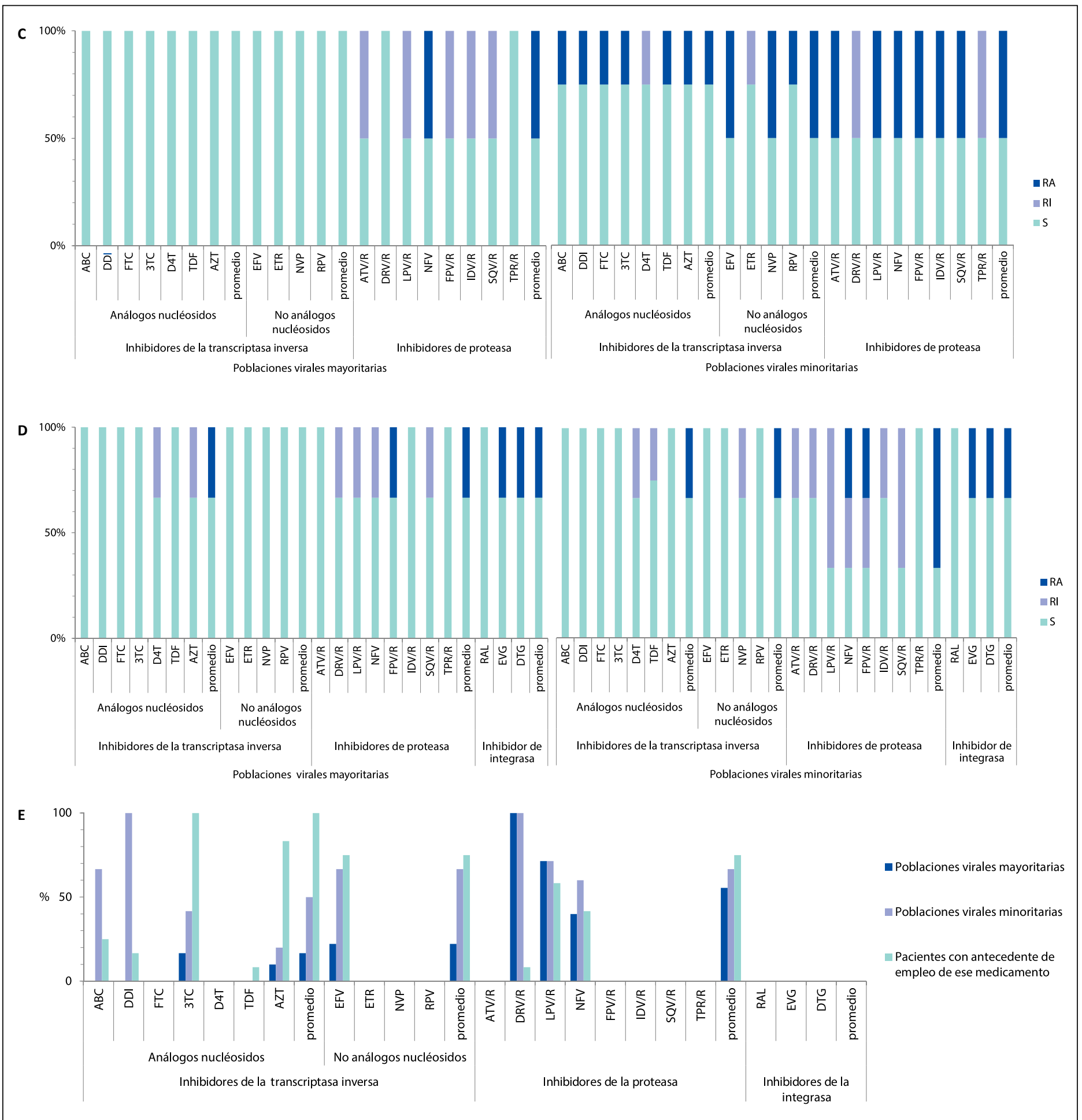

Figura 4. Porcentaje de resistencia a los antiretrovirales en poblaciones minoritarias y mayoritarias de VIH-1 según ARV utilizados previamente en pacientes niños (A: 3 esquemas terapéuticos, B: 2 esquemas terapéuticos, C: 1 esquema terapéutico, D: se desconoce cuántos esquemas terapéuticos han utilizado y E: Niveles de resistencia a los ARV y tratamiento previo con ese ARV). Abreviaturas: ABC: Abacavir, AZT: zidovudina, ATV: atazanavir, DRV: darunavir, d4T: estavudina, ddC: zalcitabina, ddl: didanosina, DTG: dolutegravir, EFV: efavirenz, ETR: etravirine, EVG: elvitegravir, FTC: Emtricitabina, FPV: fosamprenavir, IDV: indinavir, LPV: lopinavir, NFV: nelfinavir, NVP: nevirapina, RA: resistencia alta, RAL: raltegravir, RI: resistencia intermedia, RPV: rilpivirina, S: susceptible, SQV: saquinavir, TDF: tenofovir, TPR: tripanavir, 3TC: lamivudina, /R: potenciado con ritonavir. 
ambas poblaciones (datos no mostrados).

Los pacientes con LTCD4+ menores a $200 / \mathrm{mm}^{3}$ o elevados niveles de CV ( $>100.000$ copias ARN/mL) tuvieron virus con elevados niveles de resistencia para IP y para ITRNAN $(65,9$ y $59,1 \%$ para recuentos de LTCD4+ bajos y 54,3 y $43,5 \%$ para elevados niveles de $\mathrm{CV}$, respectivamente). De forma general, la mayor parte de las poblaciones virales estudiadas en la región de la TR $(76,2 \%)$ y en la región de la PR $(85,1 \%)$ fueron subtipo $\mathrm{B}$, mientras que en la región de la IN fueron subtipo no B $(54,5 \%)$, fundamentalmente CRF25_cpx $(53,4 \%)$. No se encontraron resultados de subtipos virales en las muestras provenientes de cinco pacientes en la región de la TI, en cuatro casos de la región de la PR y en 46 de la región de la $\mathrm{IN}$.

Los otros subtipos encontrados en la región de la TR fueron: CRF28_BF (8,9\%), CRF02_AG (3,0\%), CRF29_ BF y CRF12_BF (2,0\%), CRF07_BC, CRF42_BF y CRF36_cpx $(1,0 \%)$. En la región de la PR, los subtipos no B fueron CRF02_AG (4,0\%), CRF07_BC (3,0\%), subtipo F2 (2,0\%), CRF34_01B y CRF17_BF $(1,0 \%)$. En la región de la $\mathrm{IN}$ el otro subtipo no $\mathrm{B}$ detectado fue F1 $(1,0 \%)$.

Los virus tipificados, exceptuando cinco casos, fueron subtipo B o formas recombinantes con subtipo B en alguna de las regiones secuenciadas. No se encontró asociación entre los subtipos y las mutaciones encontradas $(\mathrm{p}>0,05)$.

\section{Discusión}

La pirosecuenciación permite la detección de cuasiespecies minoritarias con capacidad discriminatoria para detectar variantes virales que representan tan sólo $1 \%$ de la población ${ }^{15}$. Usando esta técnica, se han encontrado variantes de VIH resistentes a los ARV en poblaciones virales menores a $20 \%$ de aquellos pacientes con fallo a la terapia ${ }^{16}$. Las cuasi-especies pudieran emerger como poblaciones mayoritarias de virus después del inicio de la terapia de rescate en pacientes previamente tratados ${ }^{17}$.

La interpretación de las pruebas de resistencia que describen poblaciones virales minoritarias, si bien no es un criterio establecido para determinar qué medicamento ARV indicará el médico de asistencia a un paciente, puede orientar al médico sobre cuál puede ser la evolución de las poblaciones virales circulantes en el paciente y la potencialidad de desarrollar resistencia a algunos medicamentos.

Desafortunadamente, esta tecnología (pirosecuenciacion 454) fue descontinuada y solo se contó con acceso limitado a los reactivos. En la actualidad existen otras tecnologías que permiten la detección de poblaciones virales minoritarias.
En el estudio realizado se encontraron diferencias en la frecuencia de las mutaciones en las poblaciones virales estudiadas. Existe un grupo de mutaciones que son frecuentes en las poblaciones minoritarias y que, por su relevancia al relacionarse con la resistencia a determinados medicamentos, probablemente constituirá un problema para estos pacientes ${ }^{17,18}$.

Los resultados de la CV elevada y de recuentos bajos de LTCD4+ en pacientes tratados indican un fallo a la terapia de largo tiempo de evolución ${ }^{19}$. Los pacientes que porten virus con resistencia a todos los medicamentos de un grupo (FCR) o a todos ellos (MDR) en poblaciones minoritarias tendrán opciones terapéuticas seriamente comprometidas en un futuro, lo que indica un fallo de terapia de larga evolución, lo que conduce a la acumulación de mutaciones de resistencia ${ }^{14}$. El principal objetivo del tratamiento ARV es prolongar la vida de los pacientes con una calidad adecuada y al realizar pruebas de resistencia el tratamiento se enfoca de forma óptima para lograr este objetivo.

Los elevados niveles de resistencia a IP y a ITRNAN, pudieran corresponder a la alta sensibilidad de la pirosecuenciación para las poblaciones minoritarias virales, a tratamientos ARV de larga duración con estos medicamentos o a la falta de adherencia adecuada con los $\mathrm{ARV}^{20}$. Las razones más comunes del fallo virológico en pacientes con tratamientos ARV son la adherencia inadecuada a los medicamentos, la infección con otra cepa viral, la inestabilidad en el acceso a algunos medicamentos, la supresión virológica incompleta en pacientes tratados con medicamentos de baja barrera genética, los factores farmacocinéticos y la transmisión de variantes minoritarias resistentes en pacientes con fallo a la terapia de primera línea ${ }^{21}$. Debido a estas razones, es esencial determinar las causas que condujeron al fallo virológico en pacientes tratados, para poder determinar cuál será la estrategia terapéutica a seguir con estos pacientes, teniendo en consideración que el tratamiento ARV debe ser lo suficientemente potente como para suprimir los niveles de replicación viral y a su vez deben tener una barrera genética lo suficientemente alta como para evitar un rebote virológico ${ }^{21}$.

En Ecuador, no se conoce cuál ha sido el comportamiento de la resistencia, debido a que no hay estudios previos publicados. El tamaño de la muestra estudiada en la actual investigación no permite realizar una valoración de la resistencia a los ARV en pacientes con fallo a la terapia en el país y la falta de algunos datos en las fichas de datos no permite llegar a otras conclusiones. El presente estudio constituye un estudio piloto en el cual se puede apreciar algunos de los problemas relacionados con la resistencia a los ARV en una muestra de la población con VIH de Ecuador y llamar la atención de las autoridades de salud sobre la importancia de realizar de forma estable 
pruebas de resistencia a los ARV.

En Minais Gerais, Brasil, la presencia de mutaciones que confieren resistencia ha tenido un comportamiento diferente para cada grupo de ARV durante un análisis de tendencia de 10 años, aumentando para los ITRNAN, disminuyendo para los IP y manteniéndose similar para los ITRAN. En dicho estudio se encontraron predictores de resistencia a cada grupo de medicamentos diferentes ${ }^{22}$. Otro estudio en Belém, Pará, Brasil, encontró frecuencias de mutaciones mayores de $20 \%$ y hasta $80 \%{ }^{23}$. No se conoce cuál ha sido la prevalencia de la resistencia a los ARV en Ecuador, por lo que se desconoce cuál ha sido el comportamiento de ésta en el tiempo. En Europa, los pacientes con falla virológica, en 1997 eran 74,2\% y en 2012 eran sólo 5,1\%; de estas muestras, $77,9 \%$ tenía resistencia, siendo la resistencia a los ITRAN la más frecuente $(70,3 \%)^{24}$.

\section{Niveles de resistencia a los medicamentos anti-retrovirales y su relación con el tratamiento empleado}

La variedad de esquemas terapéuticos utilizados en los pacientes analizados pudiera ser considerado como un manejo terapéutico inadecuado. Entre las posibles causas de este manejo se pueden encontrar el no tener implementado estudios de resistencia para el adecuado tratamiento ARV, una incorrecta indicación médica de los ARV o las combinaciones inadecuadas de éstos, además de una pobre adherencia de los pacientes a los tratamientos.

Los pacientes estudiados con una mayor cantidad de esquemas terapéuticos tuvieron niveles de resistencia mayores. En pacientes con evidencia de fallo a la terapia, si se mantiene la misma combinación por tiempo prolongado, van acumulándose otras mutaciones que incrementan los niveles de resistencia ${ }^{19}$.

Las guías de tratamiento de la OMS en el año 2015 recomiendan como terapia de primera línea para países con bajos recursos, la combinación TDF+3TC o FTC+ EFV para adultos y adolescentes, mientras que para los niños $\mathrm{ABC}$ o $\mathrm{AZT}+3 \mathrm{TC}+\mathrm{LPV} / \mathrm{r}$ o EFV según la edad ${ }^{25}$. Estas mismas guías sugieren que como el monitoreo de la $\mathrm{CV}$ no está generalmente disponible, el primer régimen pudiera ser cambiado cuando el paciente experimente fallo inmunológico o clínico. En la "Guía de atención integral para adultos y adolescentes con infección por $\mathrm{VIH} /$ sida" de Ecuador, se recomienda como tratamiento de primera línea el $\mathrm{TDF}+\mathrm{FTC}+\mathrm{EFV}$, en concordancia con las recomendaciones de la OMS, y en caso de existir alguna contraindicación dos ITRAN + un ITRNAN o dos ITIAN + un $\mathrm{IP}^{3}$.

\section{Subtipos}

La región sudamericana ha sido considerada como una zona donde el subtipo B es mayoritario; dentro de este subtipo, la prevalencia de mutaciones que confieren resistencia es de $44,1-72 \%{ }^{26}$. La relevancia de conocer el subtipo viral es que algunos subtipos han sido relacionados con algunas vías de transmisión; por ejemplo, el subtipo B se asocia con los hombres que tienen sexo con hombres, así como algunas formas recombinantes con el uso de drogas intravenosas ${ }^{27,28}$. Además, se han descrito mutaciones que generan resistencia en algunos subtipos y no en otros (la 30 y la 90 para IP, la 106 para ITRNAN, etc. $)^{29}$. Recientemente se describió una forma recombinante con rápida progresión a SIDA, como es el caso del CRF19_cpx, presente en la epidemia cubana ${ }^{30,31}$. Las diferencias encontradas en el presente estudio dado lo pequeña y variado de la muestra no se establecieron de forma significativa.

La mayoría de los subtipos virales detectados fueron $\mathrm{B}$ y formas recombinantes de $\mathrm{B}$ en la región de la TR y la PR, lo que concuerda para el estándar de América ${ }^{30,32}$ y en la región que codifica para la IN fue el CRF25_cpx.

El subtipo CRF25_cpx está constituido por fragmentos de subtipos A, G y fragmentos sin clasificar; no existen reportes previos de una alta prevalencia de este subtipo en la región que codifica la IN. La región de la IN del subtipo CRF25_cpx se encuentra codificada por un recombinante de subtipo $G$ y fragmentos sin clasificar mayoritariamente, por lo que la amplia clasificación de este segmento de gen en CRF25_cpx se puede tratar de un artefacto del software empleado para analizar las secuencias. Estudios filogenéticos posteriores de las secuencias de estos pacientes serán necesarios para una clasificación eficiente.

\section{Niños}

El seguimiento de los niños infectados por VIH se hace más complejo, pues este grupo poblacional requiere que se analicen muestras seriadas a partir del nacimiento y de sus madres antes de comenzar el tratamiento ARV, en el parto y luego de este, además de que la adherencia depende de los padres y luego de los adolescentes. Las opciones terapéuticas en los niños son limitadas pues no todos los medicamentos tienen formulaciones pediátricas y existe el inconveniente de la transmisión de cepas resistentes de la madre al feto ${ }^{33,34}$. Los estudios de resistencia en población pediátrica son necesarios, tanto para aquellos niños tratados con falla a la terapia, como en aquellos que se comenzarán a tratar, para iniciar una terapia de primera línea altamente efectiva ${ }^{33,34}$.

Para realizar un estudio con el objetivo de conocer los mutantes virales y su asociación con los niveles de resistencias a los ARV, es necesario realizar un análisis a un mayor número de muestras, contando con los datos clínicos, terapéuticos y epidemiológicos de todos ellos. Este estudio permitiría además que se conozcan los 
subtipos y variantes genéticas del VIH-1 que circulan en Ecuador, así como su posible relación con datos epidemiológicos.

La determinación de mutaciones que confieren resistencia a los ARV es un problema que debe resolverse en todos aquellos países que incluyan dentro de sus prioridades de salud el tratamiento y control de la epidemia de VIH.

Podemos concluir que se obtuvieron los datos de resistencia a $\mathrm{ARV}$ en una población de 101 pacientes con VIH-1 en Ecuador. Se encontró un alto número de mutaciones asociadas a resistencia tanto en la PR como en la TR; lo que traduce la aparición en un futuro/presente de resistencia en estos pacientes. La forma genética causante de la infección por VIH-1 detectada en este estudio fue la $B$ en la región de la TR y la PR, mientras que para la IN fue el CRF25_cpx. El estudio confirma la importancia de la realización de las pruebas de determinación de resistencia ARV en pacientes con fallo a la terapia. El estudio de todos los pacientes con fallo a la terapia permitirá evaluar la relevancia de la frecuencia de resistencia a los ARV y hacer, en consecuencia, otras sugerencias a las autoridades de salud ecuatorianas. Se propone realizar el seguimiento de los pacientes con mutaciones en poblaciones minoritarias para evaluar la evolución de la resistencia.

Agradecimientos. A los médicos del Hospital de Infectología de Guayaquil, Tomás Rodríguez, Francisco Andino, Julián Cuesta y Dora Hasang por su invaluable ayuda en la recopilación de datos faltantes de las fichas epidemiológicas, así como en el envío de muestras de pacientes con fallo a la terapia.

\section{Resumen}

Introducción: Las recomendaciones internacionales de tratamiento anti-retroviral incluyen pruebas de resistencia para orientar el régimen de tratamiento en cada paciente, lo que no está disponible de forma estable en Ecuador. Objetivo: Describir las mutaciones que confieren resistencia a anti-retrovirales en una población de pacientes ecuatorianos. Metodología: A partir de muestras de plasma de 101 pacientes con VIH-1 con fallo a la terapia anti-retroviral, 15 niños y 86 adultos, se realizó pirosecuenciación con el GS Junior (Roche) y se analizaron las secuencias con el programa DeepChek. Resultados: Las mutaciones más frecuentes fueron M184V/I, K101E/P/H, K103N/S, D30N, M46L/I, I54L/M, V82T/F/A/S/L y L90M en adultos, y F77L, K103N/S, M46L/I, V82T/F/A/S/L y L90M en niños. Se encontró una elevada resistencia a los inhibidores de la transcriptasa reversa (TR) no análogos de nucleósidos en poblaciones minoritarias virales de adultos y niños $(34,9$ y $70 \%$, respectivamente), en los niños, tanto las poblaciones virales mayoritarias como minoritarias, fueron resistente a inhibidores de proteasa $(>45 \%)$. Los pacientes que tuvieron un mayor número de esquemas terapéuticos presentaron mayores niveles de resistencia a los anti-retrovirales. La mayoría de las muestras fueron del subtipo $\mathrm{B}$ en la región de la TR y proteasa, y CRF25_cpx en integrasa. Conclusiones: Se muestran las mutaciones y la resistencia a antiretrovirales en una población de pacientes ecuatorianos con infección por VIH-1, que permitirán realizar un llamado de alerta a las autoridades de salud sobre la necesidad de realizar estudios de resistencia.

\section{Referencias bibliográficas}

1.- Taylor S, Jayasuriya A, Smit E. Using HIV resistance tests in clinical practice. J Antimicrob Chemother 2009; 64 (2): 218-22.

2.- Aaltonen T. Estimaciones sobre el VIH y el sida (2015). 2015 [updated 2015; cited 2016 October 10]; Available from: http://www. unaids.org/es/regionscountries/countries/ ecuador.

3.- Vance C, Malo M, Armas N, Rodríguez Cadena N, Tobar R, Aguinaga G. Guía de atención integral para Adultos y adolescentes con infección por VIH-sida. Quito; 2012 [updated 2012; cited]; 114]. Available from: http://www. salud.gob.ec/guias-de-practica-clinica/.

4.- Madigan MT, Martinko JM, Stahl DA, Clark DP. Brock Biology of Microorganisms. Thirteenth ed.: Pearson; 2011.

5.- Kouanfack $C$, Montavon $C$, Laurent $C$, Aghokeng A, Kenfack A, Bourgeois A, et al. Low levels of antiretroviral-resistant HIV infection in a routine clinic in Cameroon that uses the World Health Organization (WHO) public health approach to monitor antiretroviral treatment and adequacy with the WHO recommendation for second-line treatment. Clin Infect Dis 2009; 48 (9): 1318-22.

6.- WHO definitions of clinical, immunological and virological failure for the decision to switch ART regimens. WHO; [cited 2016 December 21]; Available from: http://www.who.int/ hiv/pub/guidelines/arv2013/art/WHO_CG_ table 7.15.pdf?ua $=1$

7.- González-González M, Hermida-Álava $\mathrm{K}$, Correa-Sierra C, Gómez-Andrade L F, Machado Díaz A, Castillo-Segovia M, et al. Implementación de la pirosecuenciación en las poblaciones virales de pacientes con VIH-1 en Ecuador. Rev Cubana Med Trop 2017; 69 (2) http://www.revmedtropical.sld.cu/index.php/ medtropical/article/view/164/152.

8.- Dudley D M, Chin E N, Bimber B N, Sanabani S S, Tarosso L F. Low-Cost Ultra-wide genotyping using Roche/454 pyrosequencing for surveillance of HIV drug resistance. PLoS One 2012; 7 (5): e36494.

9.- GS Junior emPCR Amplification Method Manual Lib-A. Branford: 454 Life Sciences Corp. A Roche Company; 2012.

10.- GS Junior Sequencing Method Manual. Branford: 454 Life Sciences Corp. A Roche Company 2013.

11.- 4 Plate HIV-1 Assay Manual - Collaboration Initiative v 2.0. ROCHE, editor. Pleasanton: ROCHE; 2011.

12.- 4 Plate HIV-1 Assay Manual - Collaboration Initiative v 3.0. ROCHE, editor. Pleasanton: ROCHE; 2013.

13.- Wensing A M, Calvez V, Günthard H F, Johnson V A, Paredes R, Pillay D, et al. 2014 Update of the drug resistance mutations in HIV1. Top Antivir Med 2014; 22(3): 642-50.

14.- Vercauteren J, Deforche K, Theys K, Debruyne M, Duque L M, Peres S, et al. The incidence of multidrug and full class resistance in HIV-1 infected patients is decreasing over time (20012006) in Portugal. Retrovirol 2008; 5: 12. 
15.- Ji H, Massé N, Tyler S, Liang B, Li Y, Merksh $\mathrm{H}$, et al. HIV Drug resistance surveillance using pooled pyrosequencing. PLoS One 2010; 5 (2): e9263.

16.- Alteri C, Santoro M M, Abbate I, Rozera G, Bruselles A, Bartolini B, et al. 'Sentinel' mutations in standard population sequencing can predict the presence of HIV-1 reverse transcriptase major mutations detectable only by ultra-deep pyrosequencing. J Antimicrob Chemother 2011; 66 (11): 2615-23.

17.- Le T, Chiarella J, Simen B B, Hanczaruk B, Egholm M, Landry M L, et al. Low-abundance HIV drug-resistant viral variants in treatmentexperienced persons correlate with historical antiretroviral use. PLoS One 2009; 4 (6): e6079.

18.- Hedskog C, Mild M, Jernberg J, Sherwood E, Bratt G, Leitner T, et al. Dynamics of HIV-1 quasispecies during antiviral treatment dissected using ultra-deep pyrosequencing. PLoS One 2010; 5 (7): e11345.

19.- Gómez L F. Resistencia antirretroviral y variantes genéticas del VIH-1 en pacientes cubanos con fallo a la terapia, enero-junio de 2012. La Habana: Instituto de Medicina Tropical "Pedro Kourí"; 2012.

20.- Steegen K, Bronze M, Papathanasopoulos M A, van Zyl G, Goedhals D, Van Vuuren C, et al. Prevalence of antiretroviral drug resistance in patients who are not responding to protease inhibitor-based treatment: results from the First National Survey in South Africa. J Infect Dis 2016; 214 (12): 1826-30.

21.- Tang M W, Shafer R W. HIV-1 antiretroviral resistance: scientific principles and clinical applications. Drugs 2012; 72 (9): e1-25.

22.- Duania H, Aleixoc A W, Tupinambása U. Trends and predictors of HIV-1 acquired drug resistance in Minas Gerais, Brazil: 2002-2012. Braz J Infect Dis 2017; 21 (2): 148-54.

23.- Lopes C A F, Soares M A, Falci D R, Sprinz E. The evolving genotypic profile of HIV-1 mutations related to antiretroviral treatment in the north region of Brazil. Biomed Res Int; 2015 [updated 2015; cited 201729 agosto]; Available from: https://www.ncbi.nlm.nih.gov/ pmc/articles/PMC4620255/.

24.- Schultze A, Phillips A N, Paredes R, Battegay M, Rockstroh J K, Machala L, et al. HIV resistance testing and detected drug resistance in Europe. AIDS 2015; 29 (11): 1379-89.

25.- WHO. Policy brief: Consolidated guidelines on the use of antiretroviral drugs for treating and preventing HIV infection: What's new. Geneva; 2015 [updated 2015; cited 2016 Feb 16]; Available from: http://apps.who.int/iris/ bitstream/10665/198064/1/9789241509893 eng.pdf.

26.- Maletich Junqueira D, de Medeiros R M, Gräf T, Esteves de Matos Almeida S. Short-term dynamic and local epidemiological trends in the South American HIV-1B epidemic. PLoS One 2016; 11 (6): e0156712.

27.- Huang SW, Wang SF, Cowó ÁE, Chen M, Lin YT, Hung CP, et al. Molecular epidemiology of HIV-1 infection among men who have sex with men in Taiwan in 2012. PLoS One 2015; 10 (6): $\mathrm{e} 0128266$

28.- Cheong H T, Chow W Z, Takebe Y, Chook J B, Chan K G, Al-Darraji H A, et al. Genetic characterization of a novel HIV-1 circulating recombinant form (CRF74_01B) identified among intravenous drug users in Malaysia: Recombination history and phylogenetic linkage with previously defined recombinant lineages. PLoS One 2015; 10 (7): e0133883.

29.- Santoro M M, Perno C F. HIV-1 Genetic variability and clinical implications. ISRN Microbiol 2013; 2013: 481314. doi: 10.1155/2013/481314: Available from: http:// dx.doi.org/10.1155/2013/481314.

30.- Gómez M, Salomón H, Pando M, Kijak G, Ávila M. Distribución de subtipos y recombinantes del HIV: Situación en la Argentina. Medicina 2001; 61: 881-9.

31.- Kouri V, Khouri R, Alemán Y, Abrahantes Y, Vercauteren J. CRF19_cpx is an evolutionary fit HIV-1 variant strongly associated with rapid progression to AIDS in Cuba. EBioMedicine 2015; 2: 244-54.

32.- Aulicino P C, Holmes E C, Rocco C, Mangano A, Sen L. Extremely rapid spread of human immunodeficiency virus type $1 \mathrm{BF}$ recombinants in Argentina. J Virol 2007; 81 (1): 427-9.

33.- Pérez L, Correa C, Campos Y A, González I, Pérez J, Martínez P A, et al. Drug-resistant HIV-1 in Cuban children and their seropositive mothers. MEDICC Rev 2011; 13 (2): 24-31.

34.- Duarte H A, Harris D R, Tassiopoulos K, Leister E, Negrini S F, Ferreira F F, et al. Relationship between viral load and behavioral measures of adherence to antiretroviral therapy in children living with HIV in Latin America. Braz J Infect Dis 2015; 19 (3): 263-71. 